\title{
New aerosol delivery system for neonatal ventilator circuits
}

\author{
Mark L Everard, Julie Stammers, John G Hardy, Anthony D Milner
}

\begin{abstract}
There is mounting evidence that a variety of drugs delivered as aerosols are likely to be of benefit in neonatal units. To avoid many of the problems associated with the use of jet nebulisers in ventilator circuits, a chamber was designed to be used in conjunction with a metered dose inhaler (MDI). The dimensions $(4 \mathrm{~cm} \times 11 \mathrm{~cm})$ were chosen in an attempt to maximise drug delivery. In vitro studies were subsequently performed in order to determine the optimum operating conditions. Sodium cromoglycate delivered via this system was collected on a filter placed between the tip of an endotracheal tube and a model lung. The dose delivered was determined by means of an ultraviolet spectrophotometric assay. Using a Draeger Babylog 8000 ventilator it was found that drug delivery as maximised by actuating the device just before the inspiratory cycle when the chamber was placed adjacent to the endotracheal tube and by using a long (one second) inspiratory time. Under these conditions $1 \cdot 5-2 \%$ of the original dose was deposited upon the filter at tidal volumes of 11-22 ml. When considered in terms of body weight this is many times the equivalent dose delivered to adults from an MDI. Effective drug delivery to the filter was confirmed using a radiolabelled aerosol. Radiolabelled studies delivering aerosol to the lungs of intubated rabbits demonstrated that deposition aerosol was distributed uniformly between lobes when corrected for the weight of each lobe.

In conclusion, the device appears likely to deliver significant, reproducible quantities of drug to the lower respiratory tract while being simple to use.
\end{abstract}

Queen's Medical Centre, Nottingham Department of Child Health

Mark L Everard

Julie Stammers Anthony D Milner

Department of Medical Physics John G Hardy

Correspondence to: Dr Mark L Everard, Department of Child Health Queen's Medical Centre, Floor E, East Block. Nottingham NG7 2UH.

Accepted 14 January 1992

A simple effective system for delivery aeros to the lower respiratory tract of ventilated neonates may well prove to be a vaulable addition to the therapeutic options available in the neonatal unit. In particular, the ability to administer steroids in the form of an aerosol directly to the lower respiratory tract might prove to be extremely useful in ventilated neonates developing chronic lung cholinergic agents delivered as an aerosol have been shown to have clinical effects in ventilated and spontaneously breathing neonates. ${ }^{1-4}$ Drugs such as antioxidants and vasodilators may also be of value if they could be effectively delivered as an aerosol.

Attempts to deliver aerosols into neonatal circuits generally involve introducing jet nebulisers into the inspiratory limb of the circuit. This form of treatment has not found widespread acceptance for a number of reasons. Problems include hydroscopic growth resulting in 'raining out' within the circuit, cooling of the inspired air, great variations in nebuliser output in terms of dose delivered and size of droplets generated, and the need for a high flow rate through the nebuliser into the circuit. ${ }^{5-6}$ Additionally there is currently no cheap, satisfactory steroid formulation available for nebulisation.

The attractions of using metered dose inhalers (MDIs) include their ease of use, the ready availability of effective steroid formulations, the resistance of drug particles to hydroscopic growth due to their surfactant coating, and the optimal size distribution of drug particles once the propellants have evaporated. They have been used in ventilator circuits for a number of years. ${ }^{7-9}$ However, actuating the MDI directly into the endotracheal tube results in very considerable drug deposition on the adaptor and walls of the tubing due to direct impaction. ${ }^{9}$

To maximise drug delivery from MDIs, a chamber has been designed to be inserted into the inspiratory limb of a ventilator circuit for use with metered dose inhalers. Experiments were performed to determine the operating conditions which maximised drug delivery to the end of an endotracheal tube and to determine whether the amount of drug delivered was likely to be significant in terms of drug delivery per kg body weight. A rabbit model was used in order to determine whether an aerosol from a MDI delivered via an endotracheal tube would penetrate beyond the main bronchi in small volume lungs. This model also allowed us to assess whether aerosol deposition was likely to be distributed evenly between lobes. disease. $\beta_{2}$ Sympathomimetics and anti-

\section{Method}

IN VITRO MODEL

The chamber used in these experiments consisted of a cylindrical chamber $4 \mathrm{~cm} \times 11$ $\mathrm{cm}$ tapering at each end to connect into the tubing of the ventilator circuit. A valve stem was placed such that the valve orifice lay centrally within the proximal end of the chamber so that gas flowed around the valve stem as it entered the chamber. The device 
was introduced into the inspiratory limb of a ventilator circuit as shown in fig 1 . The ventilator used was a Draeger Babylog 8000 , which permitted monitoring of airway pressure and tidal volume. The visual display of the ventilator cycle enabled actuation of the MDI at specific points during the ventilator cycle. The endotracheal tube was connected to a model lung consisting of a rubber bellows that inflated and deflated during the ventilator cycle. Between the tip of the endotracheal tube and the model lung was placed a $1.2 \mu \mathrm{m}$ Acrodisc filter (Gelman).

Experiments were performed under the following operating conditions to assess the effect upon deposition of drug at the filter: timing of the actuation during the ventilator cycle, inspiratory time, distance of the chamber from the endotracheal tube manifold, endotracheal size, and tidal volume, 'Standard' settings of 30 breaths/minute, an inspiratory:expiratory ratio of $1: 1$, pressures of $30 / 2$, and a flow rate of $7.51 /$ minute were used initially which produced a tidal volume, when using the $1.2 \mu \mathrm{m}$ filter, of $11 \mathrm{ml}$. These experiments were repeated using an inspiratory:expiratory ratio of $1: 3$ maintaining a rate of 30 breaths per minute. In order to assess the effect of increasing the distance from chamber to endotracheal tube manifold, experiments were performed using the standard settings and actuating the MDI immediately before the inspiratory cycle when a $10 \mathrm{~cm}$ length of tubing was introduced between the chamber and manifold. Experiments were performed with both straight and corrugated lengths of tubing. A straight $3.2 \mathrm{~mm}$ internal diameter tube was used in all experiments except for one, in which a $2.7 \mathrm{~mm}$ tube was used using standard settings in order to assess the effect of tube size. To obtain larger tidal volumes, high inspiratory pressures were required and necessitated the use of a $5 \mu \mathrm{m}$ filter to achieve a tidal volume of $22 \mathrm{ml}$.

Ventilator settings used for each experiment were recorded, each experiment being per-

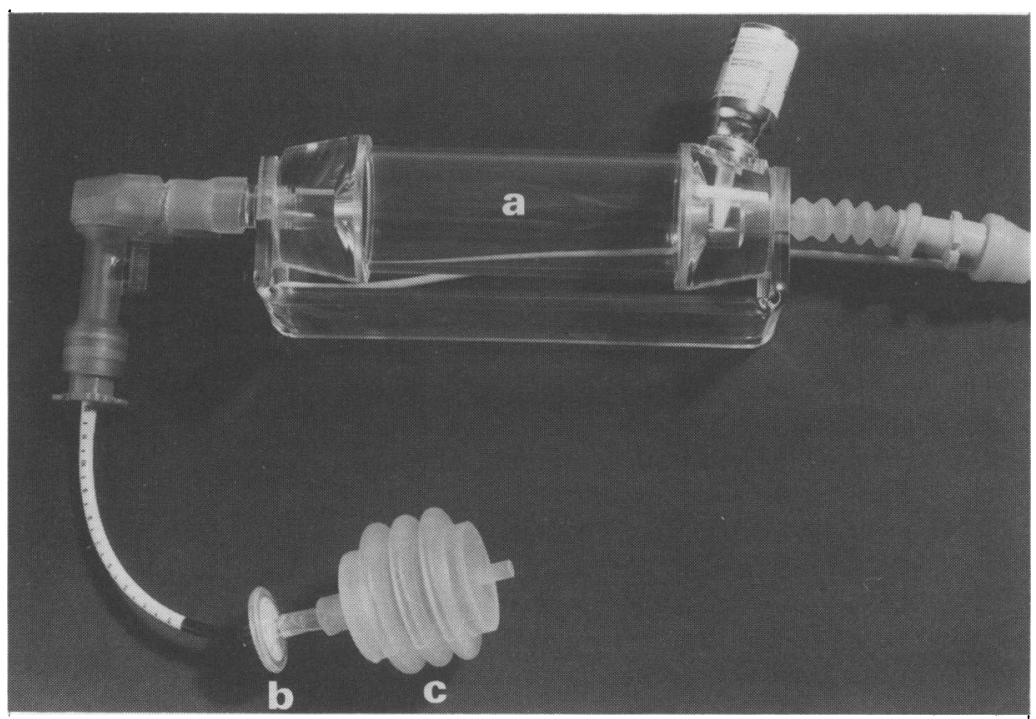

Figure 1 Chamber (a), filter (b), and model lung (c) in ventilator circuit as used in these experiments. formed four times. Results are presented as mean (SD).

DRUG ASSAY

Sodium cromoglycate was used to monitor deposition of aerosol on the filter because this drug could be assayed conveniently using a ultraviolet spectrophotometric method (Hewlett Packard Diode Array spectrophotometer). For each experiment 20 actuations of a MDI delivering $1 \mathrm{mg}$ sodium cromoglycate per actuation was used to ensure that sufficient drug was deposited on the filter to produce an accurate assay. Each actuation was separated by 30 seconds to ensure that the aerosol had cleared from the chamber.

At the end of each experiment the filter was initially washed with Freon 11 to remove surfactants from the surface of deposited drug particles. After drying, the filter was washed with $50 \mathrm{ml}$ of distilled water. The absorbance at $326 \mathrm{~nm}$ was obtained for the resultant solution and this was then used to calculate the total quantity of drug 'inhaled' per actuation using the formula:

$$
\text { Drug dose }(\mathrm{mg})=\frac{\begin{array}{c}
\text { Absorbance at } 326 \\
\mathrm{~nm} \times \text { volume }(\mathrm{ml}) \times 10
\end{array}}{\text { Exnumber of actuations }}
$$

The system had previously been calibrated using known concentrations of sodium cromoglycate, the constant $\mathrm{E}$ (absorbance for a $1 \%$ solution using a $1 \mathrm{~cm}$ path length) as 159.43. Previous experience with this method has demonstrated excellent reproducibility, this being estimated to be within $0.01 \mathrm{mg}$.

\section{RADIOLABELLING OF NEDOCROMIL SODIUM} Technetium-99m ( ${ }^{99} \mathrm{Tm}$ ) was used to radiolabel nedocromil sodium (Tilade, Fisons) contained in MDIs. Sodium pertechnetate in saline was eluted from a ${ }^{99} \mathrm{Tm}$ generator and then extracted into butanone. The solution was added to an empty MDI canister and then evaporated to dryness. The canister was cooled in liquid nitrogen, together with the MDI containing the drug to be labelled. The canisters were taken from the liquid nitrogen, the metering valve was removed from the drug-containing canister and the contents poured into that containing the ${ }^{99} \mathrm{Tm}$. A metering valve was crimped onto the canister and the labelling completed with immersion in an ultrasonic bath for 10 minutes.

Nedocromil was chosen as previous work within the department using an Andersen Sampler and multistage liquid impingers confirmed that the quantity of drug and radiolabel deposited upon each stage correlated closely.

\section{DEPOSITION OF AEROSOL WITHIN THE CIRCUIT}

To assess the pattern of deposition within the chamber a single experiment was performed with the chamber operated under standard ventilator settings and actuating the MDI immediately before the inspiratory phase. After the actuation, the equipment was 


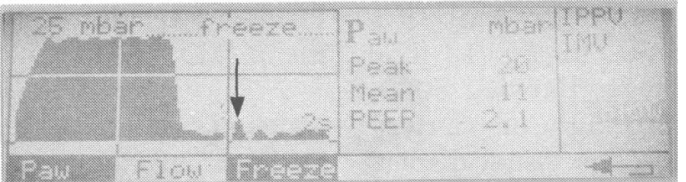

Figure 2 Visual display of Draeger Babylog 8000 ventilator showing pressure changes during one cycle of the ventilator. Pressure change due to actuation of MDI arrowed.

dismantled and the components imaged using a gamma camera. The image was displayed on a television monitor and the regions of interest defined by encompassing the image of each component. Count rates were determined, corrected for background counts, and expressed as a percentage of the total counts recorded.

\section{RABBIT PREPARATION}

Four New Zealand/Californian rabbits were studied (weight $1 \cdot 99-2 \cdot 17 \mathrm{~kg}$ ). A 24 gauge catheter was inserted into the marginal ear vein and anaesthesia induced with $5 \mathrm{ml} / \mathrm{kg}$ of $20 \%$ (w/v) ethyl carbamate (urethane, Sigma Chemical Co). A tracheostomy was per-

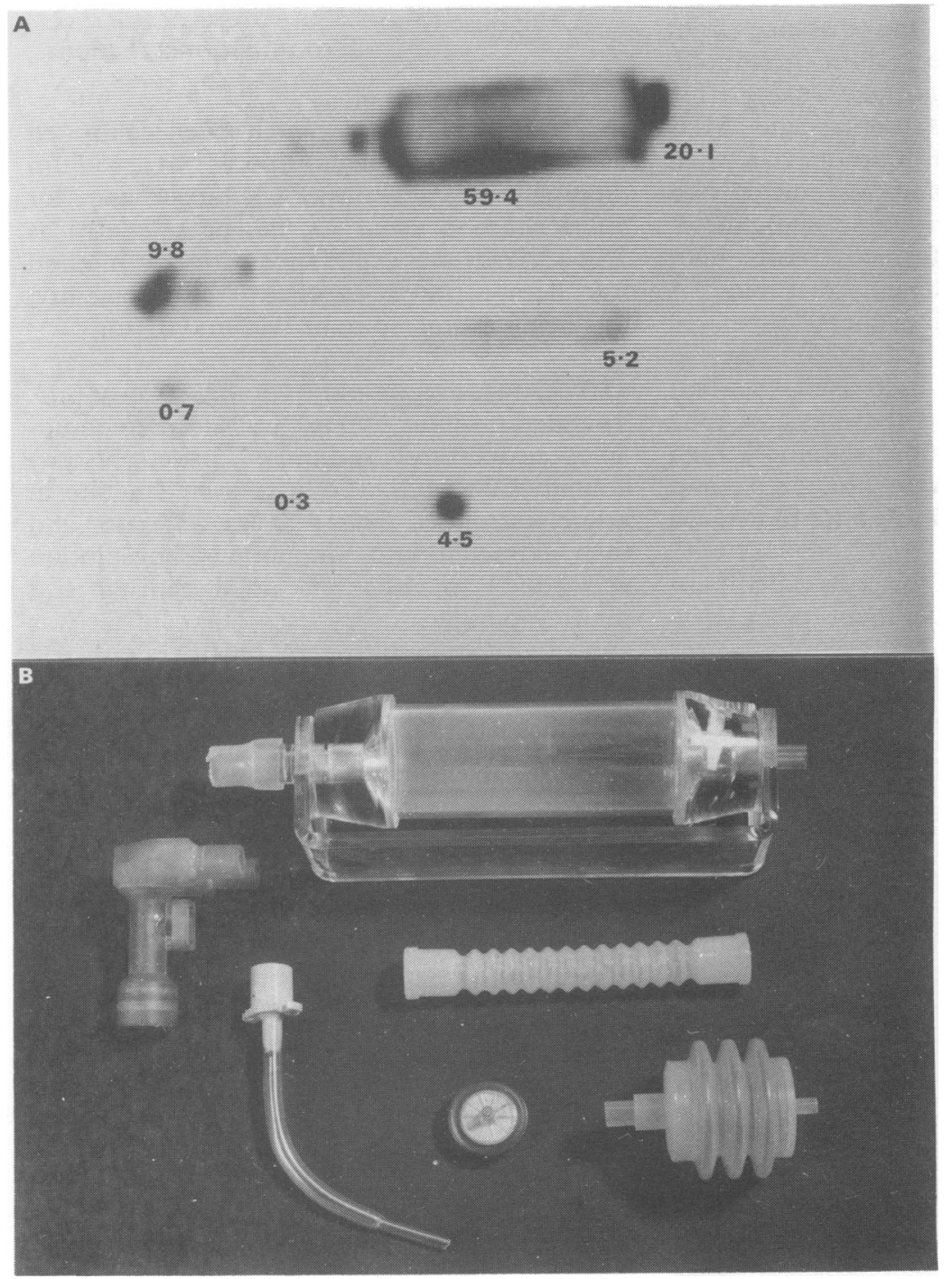

Figure 3(A) Gamma camera image of apparatus after one actuation of a radiolabelled MDI with dose deposited as a percentage of the total administered dose. (B) Apparatus as placed on gamma camera. formed and a size 3.0 endotracheal tube inserted.

ADMINISTRATION OF RABIOLABELLED AEROSOL Radiolabelled aerosol was administered via the spacing device to spontaneously breathing anaesthetised rabbits in order to determine the distribution of aerosol deposition within this animal model. It was not possible to use a ventilator circuit in these experiments and hence the chamber was connected directly onto the end of the endotracheal tube and the aerosol administered by inflating the lungs using the same technique as used in neonatal resuscitation. An air supply (4 1/minute) was connected to the distal end of the chamber by means of a $T$ piece. After actuation of the MDI, the lungs were inflated by placing a finger over the open end of the $T$ piece for approximately one second. This was repeated at one second intervals for a total of seven inflations per actuation. The pressure applied was sufficient to cause adequate chest wall expansion without causing apparent over expansion. This was repeated for a total of eight actuations per rabbit.

MEASUREMENTS OF AEROSOL DEPOSITION

Rabbits were killed immediately after aerosol administration. The lungs and trachea were dissected out and the lungs separated into individual lobes which were weighed. The amount of radioactivity in each lobe and the trachea and main bronchi were assayed for radioactivity. During each experiment the MDI containing the radiolabelled drug was actuated once into a plastic container. The count rate from the container and from the actuator were then used to calibrate the equipment for each experiment.

\section{Results}

Examination of the visual display on the Draeger Babylog 8000 ventilator (fig 2) showed that actuation of the metered dose inhaler increased the pressure within the airways by no more than 3 millibars. It is, therefore, unlikely to cause any problems in terms of the pressure generated within the circuit, particularly if it is actuated during the expiratory phase.

The effects of actuating the MDI immediately before or after the inspiratory phase are as follows. Using standard settings $1.54 \%$ $(0.05)$ of the initial dose was deposited upon the filter when the MDI was actuated immediately before the inspiratory cycle but fell to $0.83 \%(0 \cdot 10)$ when the MDI was fired immediately after the inspiratory cycle. When the inspiratory:expiratory ratio was altered to $1: 3$ at the same rate of 30 breaths/minute the dose delivered when actuating the $\mathrm{MDI}$ before and after the inspiratory cycle fell to $1.21 \%(0.02)$ and $0.24 \%(0.03)$ respectively. Introducing a $10 \mathrm{~cm}$ length of tubing between chamber and endotracheal tube manifold reduced drug deposition on the filter from $1.54 \%$ to $1 \cdot 15 \%(0 \cdot 13)$ for smooth tubing and to $0.89 \%(0.07)$ for the corrugated tubing. 
Using a $2.5 \mathrm{~mm}$ internal diameter tube rather than a $3 \mathrm{~mm}$ tube resulted in deposition upon the filter of $1 \cdot 80 \%(0 \cdot 1)$. Deposition on the filter increased from $1.54 \%$ at a tidal volume of $11 \mathrm{ml}$ to $1.87 \%(0.06)$ when the tidal volume was increased to $16 \mathrm{ml}$ and to $2.01 \%$ (0.09) with a $22 \mathrm{ml}$ tidal volume.

The gamma camera image of the radiolabelled aerosol deposition and the counts obtained from each component expressed as a percentage of the total counts in the in vitro model is shown in fig $3 \mathrm{~A}$. The apparatus as placed on the gamma camera is shown in fig 3B.

The dose reaching the lower airway and that penetrating beyond the main bronchi for each rabbit is shown in the table. The dose delivered per gram of lung tissue for each rabbit is also given. The mean dose deposited in each lobe as a percentage of the total dose penetrating beyond the main bronchi is shown in fig 4.

\section{Discussion}

The development of a simple, effective system for delivering aerosol to the lungs of ventilated neonates offers a number of potential advances in the care of ventilated preterm infants. The most useful group of drugs used in this way may prove to be steroids. These are currently used in the treatment of ventilated neonates developing bronchopulmonary dysplasia and have been shown to have short term benefits in enabling babies to be extubated. However, most studies have failed to show a long term effect on the natural history of the disease. This may relate to both the relatively late introduction of steroids, often after three or four weeks, when significant, permanent damage has already been established and to the relatively short courses used. Both of these factors have been influenced by natural concerns regarding possible adverse effects of these drugs. A single study suggested that courses longer than 42 days may beneficially influence the natural history, ${ }^{10}$ and recently the trend has been to resort to systemic steroids earlier in the hope that this may reduce the incidence of severe chronic disease. Indeed a recent report advocated the use of systemic steroids from day one of ventilation. ${ }^{11}$ The early use of steroids delivered as an aerosol may result in a reduction in the incidence of chronic lung disease while offering the advantages of delivering the drug directly to its site of action, hence minimising the potential for systemic side effects.

The chamber described in this paper was designed to maximise drug delivery at the very low tidal volume observed in the neonatal patients. Since neonatal ventilator circuits are normally of the continuous flow type, the aerosol introduced into a chamber within the circuit is likely to be diluted very rapidly during the expiratory phase of the cycle and hence it is likely that only the first breath will carry a significant bolus of aerosol. The dimensions of this chamber $(4 \mathrm{~cm} \times 11 \mathrm{~cm})$

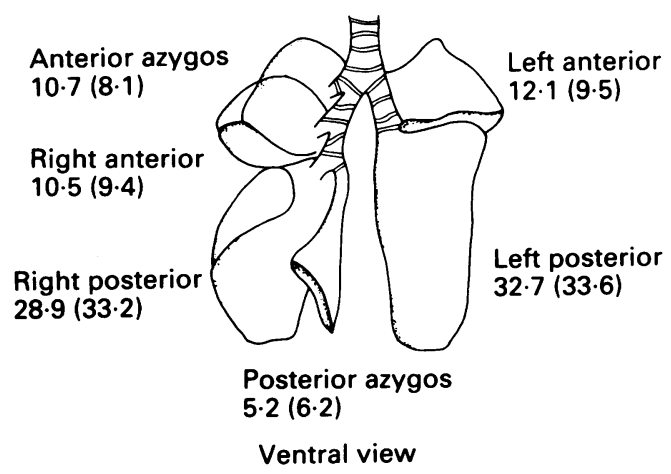

Figure 4 Mean dose delivered to each lobe expressed as a percentage of the total dose penetrating beyond the main bronchi. Mean weight of each lobe as a percentage of the total weight of the lobes is given in parentheses.

Dose of aerosol delivered to the lower respiratory tract of four rabbits as a percentage of the total administered dose

\begin{tabular}{lccccc}
\hline \multicolumn{1}{c}{1} & 2 & 3 & 4 & Mean (SD) \\
\hline $\begin{array}{l}\text { Dose deposited } \\
\text { in the trachea, } \\
\text { main brochi, } \\
\text { and lungs }\end{array}$ & 12.1 & 13.6 & 8.54 & 6.86 & $10.89(1.56)$ \\
$\begin{array}{l}\text { Dose deposited } \\
\text { in lungs }\end{array}$ & 2.35 & 5.15 & 2.71 & 2.65 & $3.22(1.30)$ \\
$\begin{array}{l}\text { Dose } \\
\text { deposited/g } \\
\text { of lung tissue }\end{array}$ & 0.29 & 0.40 & 0.24 & 0.26 & $0.29(0.07)$ \\
\hline
\end{tabular}

were chosen in order to maximise the aerosol concentration within the chamber ${ }^{12}$ and hence maximise drug delivery during the first inspiratory cycle. An additional benefit of using a small chamber is that it will be much easier to handle. A similar chamber devised independently has been used to deliver radiolabelled aerosol to ventilated adults. ${ }^{13}$ In this study, using a volume preset time cycled ventilator, it was found that approximately $5.5 \%$ of the dose from a MDI delivered via the extension chamber was deposited into the lungs, compared with only $1 \cdot 2 \%$ from a jet nebuliser. Using a dosimetry technique timed with inspiration, delivery from jet nebulisers to the lungs of ventilated adults can be significantly increased. ${ }^{14}$ However, such systems are likely to be relatively expensive and the other disadvantages of using jet nebulisers still apply. Drug delivery from a chamber such as the one described in this paper can be simply increased by further actuations of the metered dose inhaler.

Placing a chamber such as this in the inspiratory limb of the circuit rather than placing it between the endotracheal tube and the manifold as proposed by some workers will reduce drug delivery but will minimise the disturbance experienced by the neonate. Hence it may be possible to use such a device safely with acutely ill infants early in the course of the respiratory distress syndrome. The results presented here suggest that significant drug delivery can be obtained when used in this way, while the absolute dose delivered can be easily increased simply by increasing the number of actuations. These results suggest that drug delivery can be maximised by actuating the MDI immediately 
before the inspiratory cycle and using a longer inspiratory period. Others have described increased deposition in animal experiments when using longer inspiratory times and postulated that the longer residence period may increase deposition in the lower respiratory tract by sedimentation. ${ }^{15}$ However, sedimentation is not likely to be a significant factor in the present experiments and hence some other effect, possibly related to the pattern of flow, may be operating. The surprisingly large drop in drug deposition at the filter when using $10 \mathrm{~cm}$ of smooth tubing may relate more to the timing of the actuation of the MDI. Actuating it immediately before the inspiratory phase may mean that the leading edge of the aerosol bolus has not reached the endotracheal tube manifold. More sophisticated timing of the actuation directly from the ventilator is necessary before the relationship between chamber position and actuation is further explored. The fall in drug delivery when using a smaller tube is a little surprising in view of the minimal deposition within the endotracheal tube noted on the gamma camera image. However, similar trends have been described in experiments delivering aerosols from MDIs when using different sizes of adult endotracheal tubes. ${ }^{9}$

The doses delivered to the filter may appear to be very small, but are in fact extremely large doses when considered in terms of body weight. Adults using a good inhaler technique may achieve drug delivery to the lower respiratory tract of perhaps $10 \%$ of the original dose delivered by the MDI, a $11 \mathrm{ml}$ tidal volume might represent that of a $1 \mathrm{~kg}$ baby who would require only $0 \cdot 14 \%$ of the original dose to receive the same dose on a per kilogram basis as a $70 \mathrm{~kg}$ adult. In all these experiments, even those under suboptimal conditions, the drug delivered to the filter was in excess of this dose.

The rabbits' experiments show significant variations in delivery of aerosol to the lower respiratory tract when using a method of inflating the lungs similar to that used in neonatal resuscitation. Drug delivery is inevitably higher than in the in vitro studies as seven inflations were used. When used in this way the spacing chamber also acts as a holding chamber. The variation in deposition within the trachea and bronchi is considerable and may relate to factors such as the rabbit's respiratory phase during actuation and to whether the short tracheostomy tube was straight or curved at the time of actuation. The variation in dose penetrating beyond the trachea and bronchi was less pronounced while dose delivered per gram of lung tissue was remarkably consistent. The average combined weight of both lungs in an adult male is approximately $850 \mathrm{~g}^{16}$ so that the dose delivered to the lungs of these rabbits represents more than 40 times the dose per gram of lung that may be delivered when an adult patient uses the MDI.
Distribution of aerosol to each lobe was remarkably even when correlated for the weight of each lobe despite the supine posture of the rabbits. This suggests that this form of treatment is relatively free of the gravitational effects observed when liquids are introduced into the airways via endotracheal tubes. These results, however, were obtained in animals with healthy lungs; distribution within the lower respiratory tract in clinical practice will be greatly influenced by parameters beyond the clinician's control such as ventilation defects and excessive secretions.

In conclusion, effective drug delivery to the lower respiratory tract of ventilated neonates is possible when using aerosols from MDIs delivered by a cheap, simple to use, small volume chamber introduced into the inspiratory limb of a neonatal ventilator circuit. Drug delivery may be maximised by using longer inspiratory times and by actuating the MDI immediately before the inspiratory phase when the chamber is placed close to the endotracheal tube manifold. The optimal timing if used at distances from the manifold needs to be determined. The distribution of aerosol deposition appears to be relatively uniform in healthy lungs, the dose reaching each lobe in the rabbit model correlating closely with its weight.

Dr Everard was supported by Asthma Nationwide.

1 Gomez-Del Rio M, Gerhardt T, Hehre D, Feller R, Bancalari $E$. Effect of beta-agonist nebulisation in neonates with increased pulmonary resistance. Pediatr

2 Wilkie RA, Bryan MH. Effects of bronchodilators on airway resistance in ventilator-dependent neonates with chronic lung disease. $f$ Pediatr 1987;111:278-82.

3 Motoyama EK. Fort MD, Klesh KW, Mutich RI, Guthrie RS. Early onset of airway reactivity in premature infants with bronchopulmonary dysplasia. Am Rev Respir Dis 1987;136:50-7.

4 Kao LC, Durand DJ, Nickerson BG. Effects of inhaled metaproterenol and atropine on the pulmonary mechanics of infants with bronchopulmonary dysplasia. Pediatr Pulmonol 1989;6:74-80.

5 Silverman M. Aerosol therapy in the newborn. Report of a workshop. Arch Dis Child 1990;65:906-8.

6 Cameron D. Clay MM Silverman M Evaluation of nebulisers for use in neonatal ventilator circuits. Crit Care Med 1990;18:866-70.

7 Bush G. Aerosol delivery devices for the anaesthetic circuit. Anesthesiology 1986;65:240.

8 Crogan SJ, Bishop MJ. Delivery efficiency of metered dose aerosols given via endotracheal tubes. Anesthesiology 989;70:1008-10.

9 Gay PC, Hermant GP, Nelson SB, Gillies B, Hubmayer RD. Metered dose inhalers for bronchodilator delivery in intubated, mechanically ventilated patients. Chest 1991;99:66-71

10 Cummings JJ, D'Eugenio DB, Gross SJ. A controlled trial of dexamethasone in preterm infants at high risk for bronchopulmonary dysplasia. $N$ Engl f Med 1989;320: 1505-10.

11 Yeh TF, Torre JA, Rastogi A, Anyebuno MA, Pides RS. Early postnatal dexamethasone therapy in premature infants with severe respiratory distress syndrome: double-blind, controlled study. $\mathcal{f}$ Pediatr 1990;117
273-82.

2 Everard ML, Clark AR, Milner AD. Drug delivery from holding chambers with attached facemask. Arch Dis Child 1992;67:580-5.

13 Fuller HD, Dolovich MB, Posmituck G, Wong Pack W, Newhouse MT. Pressurized aerosol versus jet aerosol Newhouse MT. Pressurized aerosol versus jet aerosol
delivery to mechanically ventilated patients. Am Rev

14 Dahlback M, Wollmer P, Drefeldt B, Konson B. Controlled aerosol delivery during mechanical ventilation. Controlled aerosol delivery during mechan

15 fournal of Aerosol Medicine 1989;2.339-47. delivery in neonatal ventilator circuits. Pediatr Pulmonol delivery in neon

16 Symmers WStC, ed. The lungs. Vol 5 of Systemic pathology. Corrin B, ed. London: Churchill Livingstone, 1990. 